\title{
Structural and optical properties of Yttrium Oxide thin films for planar waveguiding applications
}

\author{
Stuart J. Pearce, ${ }^{\text {a) }}$ Greg J. Parker, ${ }^{\text {a) }}$ Martin D.B. Charlton, ${ }^{\text {a) }}$ \\ and James S. Wilkinson ${ }^{\text {b) }}$ \\ a) Electronic and Computer Science, Southampton University, SO17 1BJ \\ b) Optoelectronics Research Center, Southampton University, SO17 1BJ
}

Thin films of Yttrium Oxide, $\mathrm{Y}_{2} \mathrm{O}_{3}$ were deposited by reactive sputtering and reactive evaporation to determine their suitability as a host for a rare earth doped planar waveguide upconversion laser. The optical properties, structure and crystalline phase of the films were found to be dependent on the deposition method and process parameters. X-ray diffraction (XRD) analysis on the 'as-deposited' thin films revealed that the films vary from amorphous to highly crystalline with a strong peak along the $<222>$ plane of $\mathrm{Y}_{2} \mathrm{O}_{3}$. The samples with the polycrystalline structure had a stoichometry close to bulk cubic $\mathrm{Y}_{2} \mathrm{O}_{3}$. SEM imaging revealed a regular column structure confirming their crystalline nature. The thin film layers which allowed guiding in both the visible and infra-red region had lower refractive indices, higher oxygen content and had a more amorphous structure. Higher oxygen pressures during the deposition leads to a more amorphous layer.

PACS numbers: 68.55. J-, 78.66. -w, 81.15. -z, 42.82.Et, 78.20. -e 


\section{INTRODUCTION}

Recently there has been considerable interest in fabrication and characterization of waveguide layers as solid-state laser host materials for use as optical amplifiers in telecommunications. These include Aluminum Oxide, ${ }^{1}$ Zirconium Oxide, ${ }^{2}$ Titanium Oxide, ${ }^{3}$ Scandium Oxide, ${ }^{4}$ and Yttrium Oxide. ${ }^{5-7}$ Planar waveguide lasers made with rare earth doped thin films are a desirable method to achieve high optical gain in a small and compact device. ${ }^{5}$

In selecting the most suitable material for a rare earth doped solid state waveguide laser, desirable properties for a host material include: low optical loss, low peak phonon energy, ability to be easily doped, and ability to waveguide at the pump and emission wavelengths. Among the available host materials, the dielectric Yttrium Oxide $\left(\mathrm{Y}_{2} \mathrm{O}_{3}\right)$ has received much attention in recent years due to its excellent material properties and its suitability as a waveguide and a laser host. ${ }^{8}$ Its material properties include a high refractive index $(1.7-1.9),{ }^{9,10,11}$ large band gap, excellent thermal conductivity, a low dominant phonon energy, ${ }^{12,13}$ a broad transparency range and can be easily doped with rare earth ions such as Erbium. ${ }^{6}$ An advantage of using Yttrium Oxide as the host material for Erbium is its crystal structure. Both Yttrium Oxide and Erbium Oxide have a cubic crystal structure with very similar lattice constants and trivalent Yttrium and Erbium have nearly the same ionic radius ${ }^{5,14}$ making doping easier due to very little lattice mismatch between the two materials. One disadvantage of Yttrium Oxide for waveguide applications is its tendency to crystallize which can lead to high waveguide losses in the visible region. ${ }^{15}$ However, the crystallized structure can be advantageous to other applications such as replacing $\mathrm{SiO}_{2}$ for high $k$ gate dielectric layers. ${ }^{16}$

Another area of interest for rare earth doped materials is the ability to convert from infrared to visible wavelengths using an upconversion process. Upconversion presents an interesting route to produce visible lasers based on rare earth transitions pumped directly by cheap infra-red semiconductor lasers. Erbium doped materials exhibit strong fluorescence and are very efficient in the visible as well as in the infrared region. ${ }^{17}$ The visible fluorescence is caused by an upconversion process and is an undesirable effect in standard $1.5 \mu \mathrm{m}$ telecommunication applications. ${ }^{5}$ However, this upconversion process can be utilized in the fabrication of simple, compact lasers operating in the visible region. By replacing the erbium ions with other rare earth ions such as Thulium, ${ }^{18-20}$ Praseodymium, ${ }^{21,22}$ or Holmium ${ }^{20,23}$ upconversion lasers that emit at a range of visible wavelengths can be developed. ${ }^{24}$

For the upconversion process to be efficient the maximum phonon energy of the host material needs to be low to reduce the probability of radiationless phonon transitions. $^{25}$ These non-radiative transitions shorten the lifetime of the higher energy level reducing the probability of upconversion. The aim of the present study is to report on the structural and optical properties of thin $\mathrm{Y}_{2} \mathrm{O}_{3}$ layers produced using standard thin film deposition methods for use as a host material for a rare earth doped planar waveguide upconversion laser. 


\section{WAVEGUIDE PREPARATION}

Thin films of $\mathrm{Y}_{2} \mathrm{O}_{3}$ were prepared using high purity (99.99\%) cubic $\mathrm{Y}_{2} \mathrm{O}_{3}$ using an electron beam deposition system (LAB700, Leybold Optics) in an oxygen atmosphere. Oxygen was introduced into the chamber during the deposition to oxidize Yttrium Oxide or Yttrium dissociated by the electron beam bombardment. The oxygen partial pressure estimated from the oxygen flow introduced in to the chamber varied from $3 \times 10^{-4}$ mbar to $8 \times 10^{-5}$ mbar (high $\mathrm{O}_{2}$ flow to low $\mathrm{O}_{2}$ flow). Using a mass flow controller and pressure monitor, the process pressures were maintained during the evaporation. Before deposition the chamber was pumped down using a cryogenic pump to a starting pressure in the order of $10^{-6}$ mbar. The deposition rates in the LAB700 were monitored using a quartz crystal oscillator.

Films were deposited on to unheated $<100>$ orientated silicon substrates with either a reactively sputtered silicon dioxide buffer of $1.2 \mu \mathrm{m}$ thick or a thermally grown $2 \mu \mathrm{m}$ thick $\mathrm{SiO}_{2}$ layer. The $\mathrm{SiO}_{2}$ layer enabled a slab waveguide to be created for the purpose of optical transmission measurements. Films were also deposited using ionbeam assisted deposition (IBAD) in the LAB700, (sample d), another electron beam deposition system (Evatec BAK550), (sample e), and using reactive sputtering with oxygen in a standard high target utilization sputtering system (HiTUS) (samples $f$ and g) to allow comparison. In IBAD, evaporated material and intense plasmas are directed simultaneously to the substrate to improve stoichometry of the deposited thin films. The ion gun was ignited using an automatic process and once stable $25 \mathrm{sccm}$ of $\mathrm{O}_{2}$ was used with a discharge and bias current of $5 \mathrm{Amps}$ to produce the high-density plasma. The HiTUS technology has the benefit that the target voltage is independent of the ion density, thereby offering a wide processing space, which can be used to tailor film properties. Sputtering was achieved using a high-density plasma in conjunction with a 4 inch diameter DC biased yttrium target operating in constant power mode. Prior to deposition, the substrates were cleaned using a low intensity plasma to improve adhesion and remove any surface contamination. Table 1 gives the process parameters for each of the samples.

\section{CHARACTERIZATION RESULTS AND DISCUSSION}

Spectroscopic ellipsometry was used to determine the optical properties of the asdeposited thin films. Figure 1 shows the dependence of the refractive indices as a function of the wavelength of the incident light (material dispersion) for the $\mathrm{Y}_{2} \mathrm{O}_{3}$ films given in Table 1. The index of the $\mathrm{Y}_{2} \mathrm{O}_{3}$ thin films varied from 1.66 up to 1.92 depending on the deposition parameters.

To test the transmission properties of the $\mathrm{Y}_{2} \mathrm{O}_{3}$ deposited films, substrates were cleaved into pieces and the tunable signal output from a Coherent OPA 9400 laser, tuned to $510 \mathrm{~nm}$ was coupled into the slab by end fire coupling using a $3 \mathrm{~mm}$ focal length anamorphic lens. Samples were also tested with an infra-red laser operating at $980 \mathrm{~nm}$. Although the samples were not polished to an optical finish, the cleaved facets were of good enough quality to allow strong coupling into the slab waveguide. We found that all samples guided well at $980 \mathrm{~nm}$ over distances of $>3 \mathrm{cms}$ but only samples (a), (b) and (c) guided in the visible, over the same distance. However, sample $(\mathrm{g})$ also guided at $510 \mathrm{~nm}$ across a very short distance $(<1 \mathrm{~cm})$. One of the 
important characteristics of any waveguiding layer is its propagation loss. Losses in thin waveguide layers originate from many factors ${ }^{26}$, which include absorption in the core, leakage between the core and the cladding and scattering losses. One of the key contributors to scattering loss are the sizeable grain boundaries between neighboring crystal domains, which lead to strong Rayleigh scattering of confined light out of the waveguide. The intensity of scattered light is proportional to the Rayleigh scattering cross section which scales as $\lambda^{-4} \times \mathrm{R}^{-2}$, (where $\mathrm{R}$ is radius of the scattering particle). Hence waveguide loss can be minimized by optimizing deposition parameters to deposit a highly dense, but amorphous layer, with no sizeable grain boundaries and small grain size.

Samples with refractive index much lower than bulk cubic $\mathrm{Y}_{2} \mathrm{O}_{3}(1.934 @ 550 \mathrm{~nm})$ i.e. (a), (b), (c), and (g) demonstrated guiding at visible wavelengths, whereas samples with a high refractive index similar to the bulk value did not. This was attributed to the structure of the thin films. An amorphous layer will have a lower refractive index than a crystalline layer due to the difference in densities between them. This could be attributed to a uniform inclusion of nanometer scale air voids. ${ }^{27,28}$ Furthermore at high pressures the low kinetic energy of the evaporated material prevents the crystalline growth and leads to an amorphous films. ${ }^{29}$ Another interesting point to note is that introduction of oxygen into the chamber reduces the growth rate by slowing the evaporated atoms within the plasma. Thin films produced under these conditions can exhibit a lower refractive index. ${ }^{6,9,10}$

The phonon energy of the as-deposited $\mathrm{Y}_{2} \mathrm{O}_{3}$ was measured using normal incident Raman spectroscopy and compared with the spectra using the bulk evaporation material at $532 \mathrm{~nm}$ and $633 \mathrm{~nm}$ wavelengths. The Raman spectra from sample (a) gave a maximum phonon energy of $370 \mathrm{~cm}^{-1}$, which is in good agreement with the phonon energy of the bulk material $\left(377 \mathrm{~cm}^{-1}\right){ }^{12,13}$

The structure and crystallinity of all the samples was investigated by X-ray diffraction using the $\mathrm{Cu} \mathrm{K} \alpha$ radiation from a Bruker D8 XRD system operating at a voltage and current of $40 \mathrm{kV}$ and $40 \mathrm{~mA}$ respectively. The diffractrograms were collected in the range of $5-60^{\circ}$ using asymmetrical diffraction geometry at a fixed grazing incidence angle of $2^{\circ}$ so more of the sample could be excited. Figure 2 shows the XRD patterns of the seven $\mathrm{Y}_{2} \mathrm{O}_{3}$ samples deposited using the different methods. The diffractograms all show a small broad peak at $\sim 29^{\circ}$ which corresponds to reflections from the $<222>$ planes of $\mathrm{Y}_{2} \mathrm{O}_{3}$ [7]. The cubic phase of $\mathrm{Y}_{2} \mathrm{O}_{3}$ is expressed primarily by reflections from the $<222>$ planes. ${ }^{8,30}$ Sample (a) shows a weak and very broad diffraction peak suggesting that the layer is close to being amorphous, with further optimization a mainly amorphous structure may be possible. Samples (d) and (f) show reflections of the $\langle 222\rangle,<400\rangle,<440\rangle$, and $<622>$ planes suggesting the films are the cubic polycrystalline phase of $\mathrm{Y}_{2} \mathrm{O}_{3} .{ }^{30,31}$

The stoichometry of the thin films were then analyzed by Energy Dispersive X-ray (EDX) spectrometry. The EDX measurement was performed in a Carl Zeiss EVO SEM using INCA EDX software supplied by Oxford Instruments. Due to the large penetration depth of EDX each sample scan included a signature peak of the substrate (Si) and buffer layer $\left(\mathrm{SiO}_{2}\right)$, which caused an error in the calculation of the atomic weight percentage for the core layer. However, the INCA software allows for a degree of manipulation as the layer of interest can be separated from the substrate and 
buffer layer so that the core layer could be analyzed separately. Figure 3 shows the atomic percentage for each sample. The EDX analysis shows that samples (d), (e) and (f) are closer to the theoretical atomic weight percentage of $\mathrm{Y}_{2} \mathrm{O}_{3}(60 \%$ Oxygen, $40 \%$ Yttrium) and is consistent with a higher refractive index. Samples (a), (b), (c), and (g) have a higher oxygen content confirming the lower refractive index and the density.

Samples (b), (e) and (f) were subsequently gold coated and loaded into a JEOL 7500 Field Emission Scanning Electron Microscopy (FESEM) so the morphology and crystal structure could be investigated. Figure 4 shows the images for the samples. The SEM images confirm the XRD results for the thin films. The SEM image for sample (f) (Figure 4(iii)) shows clearly the regular columnar structure expected in a crystalline material. The SEM image of sample (b) (Figure 4(i)) shows a rough surface without the column features suggesting a more amorphous layer.

\section{CONCLUSIONS}

The relationship between the optical and structural properties and the method of deposition of $\mathrm{Y}_{2} \mathrm{O}_{3}$ thin film layers were investigated with the view to using the layers as the host for a rare earth doped planar waveguide upconversion laser. Using X-ray diffraction, spectroscopic ellipsometry and energy dispersive $\mathrm{X}$-ray spectroscopy the as-deposited films were characterized. The tendency of $\mathrm{Y}_{2} \mathrm{O}_{3}$ to form polycrystalline material was observed in several of the samples using X-ray diffraction. These samples had a stoichometry close to bulk Yttrium Oxide and SEM imaging revealed a regular column structure confirming the crystalline nature. The guiding properties of each sample were examined in the infra-red and visible region. The results indicated that the thin film layers which guided at both wavelengths had the higher oxygen content relative to the theoretical atomic weight percentage of $\mathrm{Y}_{2} \mathrm{O}_{3}$. The higher oxygen content decreases the refractive index of the layer producing a more amorphous layer and allows guiding in both the visible and infra-red. These properties make these thin film layers a promising host material for a planar upconversion waveguide laser.

\section{ACKNOWLEDGEMENTS}

The authors would like to thank Dr Sarah Thornley at Plasma Quest Limited for supplying the HiTUS $\mathrm{Y}_{2} \mathrm{O}_{3}$ thin films and MIR Enterprise for electron beam evaporation thin films for comparison. We also express our gratitude to the EPSRC (Grant Number: EP/G003319/1) for funding this research. 


\section{REFERENCES}

${ }^{1}$ C. Strohhofer and A. Polman, Opt. Mater., 21, (2003), 705

${ }^{2}$ R. Schermer, W. Berglund, C. Ford, R. Ramberg, and A.Gopinath, , IEEE J

Quantum Electron., 39, (2003), 154

${ }^{3}$ A. Bahtat, M. Bouderbala, M. Bahtat, M. Bouazaoui, J. Mugnier, M. Druetta, Thin Solid Films, 323, (1998), 59

${ }^{4}$ Y. Kuzminykh, A. Kahn and G. Huber, Opt. Mater., 28, (2006), 883

${ }^{5}$ A. Peeva, A. Og.Dikovska, P. A.Atanasov, M. Jimenez de Castro, W. Skorupa, App. Surf. Sci., 253, (2007), 8165

${ }^{6}$ O. Pons Y Moll, A Huignard, E. Antic-Fidancev, P. Aschehoug, B. Viana, E. Millon, J. Perriere, C. Garapon, J. Mugnier, J. Lumi., 87-89, (2000), 1115

${ }^{7}$ M.B.Korzenski, Ph.Lecoeur, B. Mercey, P. Camy and J.L. Doualan, App. Phys. Lett., 78, (2001), 1210

${ }^{8}$ S. Zhang and R. Xiao, J. App. Phys., 83, (1998), 3842

9 R.J. Gaboriaud, F. Pailloux, P.Guerin and F.Paumier, J.Phys. D: Appl. Phys. 33 (2000), 2884

${ }^{10}$ A. Kasikov, Appl. Surf. Sci. 254, (2008), 3677.

${ }^{11}$ T.T. Van and J. P Chang, Appl. Phys. Letts. 87, (2005), 011907

${ }^{12}$ H. Guo and Y. M. Qiao, Opt Mater., 31, (2009), 583

${ }^{13}$ A. Ubaldini and M.M. Carnasciali, J. Alloys Compd., 454, (2008), 374

${ }^{14}$ A. Polman, J. Appl. Phys., 82, (1997), 1

${ }^{15}$ B.J.H. Stadler and M. Oliver, J. App. Phys., 84, (1998), 93

${ }^{16}$ R.J. Gaboriaud, F.Paumier, F. Pailloux and P.Guerin, Mater. Sci. Eng. B, 109 (2004) 34.

${ }^{17}$ R. Kapoor, C.S. Friend, A.Biswas and P.N. Prasad, Opt. Lett., 25 (2000), 338

${ }^{18}$ T. Herbert, R Wannemacher, R. M. Macfarlane and W. Lenth, Appl. Phys. Lett 60 (1992), 2592

${ }^{19}$ S.Guy, D.P. Shepherd, M.F.Joubert, B. Jacquier and H.Poignant, J. Opt. Soc. Am. B 14 (1997) 926

${ }^{20}$ B. Peng and T. Izumitani, Opt. Mats. 4 (1995) 701

${ }^{21}$ D.M.Baney, G. Rankin and K-W.Chang, Opt. Letts. 21, (1996), 1372

${ }^{22}$ A.C.Tropper, J.N.Carter, R.D.T.Lauder, D.C.Hanna, S.T.Davey and D.Szebesta, J.Opt. Soc. Am. B, 11, (1994), 886

${ }^{23}$ V.Lavin, F.Lahoz, I.R.Martin, U.R.Rodriguez-Mendoza, J.M.Cáceres, Opt. Mats., 27, (2005), 1754

${ }^{24}$ H Eilers, Mater. Lett., 60, (2006), 214

${ }^{25}$ C.B.Layne, W.H.Lowdermilk and M.J.Weber, Phys. Rev. B, 16, (1977), 10

${ }^{26}$ Y.S.Kim and W.H Kim, Opt. Mater., 14, (2000), 229

${ }^{27}$ F.K.Shan, G.X.Liu, W.J.Lee, G.H.Lee, I.S.Kim, B.C.Shin, Int. Ferroelectrics, 80, (2006), 197

${ }^{28}$ W. Qiu, Y.M.Kang and L.L.Goddard, Appl. Phys. Letts, 96, (2010), 141116

29 O. Pons-Y-Moll, J.Perriere, E. Millon, R. M. Defourneau, D. Defourneau, B. Vincent, A. Essahlaoui, A Boudrioua and W. Seiler, J. Appl. Phys., 92, (2002), 4885.

${ }^{30}$ E.K.Evangelou, C. Wiemer, M Fanciulli, M.Sethu and W Cranton, J. App. Phys., 94, (2003), 318

${ }^{31}$ T. Tsutsumi, Jpn. J. App. Phys., 9, (1970), 735 
Table 1. Process parameters for each of the samples produced.

\begin{tabular}{|c|c|c|c|c|c|c|c|}
\hline Sample & (a) & (b) & (c) & (d) & (e) & (f) & (g) \\
\hline $\begin{array}{l}\text { Process Pressure } \\
\text { (mbar) }\end{array}$ & $3 \times 10-4$ & $1 \times 10-4$ & $8 \times 10-5$ & $2 \times 10-4$ & $1 \times 10-4$ & $2.7 \times 10-3$ & $\begin{array}{l}1.1 \times 10-2 \\
\text { (Baffled) }\end{array}$ \\
\hline $\begin{array}{c}\text { Deposition rate } \\
(\mathrm{nm} / \mathrm{s})\end{array}$ & 0.5 & 0.5 & 0.5 & 1 & 0.5 & 1.05 & 0.37 \\
\hline $\begin{array}{c}\mathrm{O}_{2} \text { flow rate } \\
(\mathrm{sccm})\end{array}$ & $\sim 70$ & $\sim 25$ & $\sim 10$ & $\sim 25$ & - & 9 & 5 \\
\hline $\begin{array}{c}\text { Thickness of buffer } \\
\text { layer }(\mu \mathrm{m})\end{array}$ & 1.2 & 1.2 & 1.2 & 1.2 & 2 & 2 & 2 \\
\hline $\begin{array}{l}\text { Thickness } \\
\text { (nm) }\end{array}$ & 474 & 461 & 490 & 510 & 455 & 460 & 458 \\
\hline Deposition method & e-beam & e-beam & e-beam & IBAD & e-beam & HiTUS & HiTUS \\
\hline $\begin{array}{l}\text { Refractive Index } \\
\text { (a) 550nm }\end{array}$ & 1.718 & 1.729 & 1.741 & 1.911 & 1.917 & 1.868 & 1.660 \\
\hline
\end{tabular}

\section{LIST OF CAPTIONS}

Fig. 1. Dispersion curves for the $\mathrm{Y}_{2} \mathrm{O}_{3}$ thin films deposited using different parameters.

Fig. 2. XRD patterns at an incidence angle of $2^{\circ}$ for the $\mathrm{Y}_{2} \mathrm{O}_{3}$ thin films deposited using different parameters.

Fig. 3. Thin film composition for each sample measured using EDX.

Fig. 4. SEM photographs of $\mathrm{Y}_{2} \mathrm{O}_{3}$ thin film layers (i) sample (b), (ii) sample (e) and (iii) sample (f). 


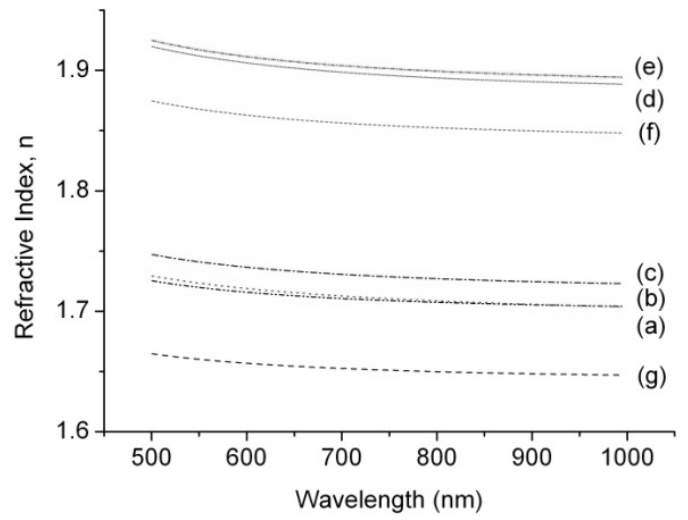

Fig. 1. Dispersion curves for the $\mathrm{Y}_{2} \mathrm{O}_{3}$ thin films deposited using different parameters.

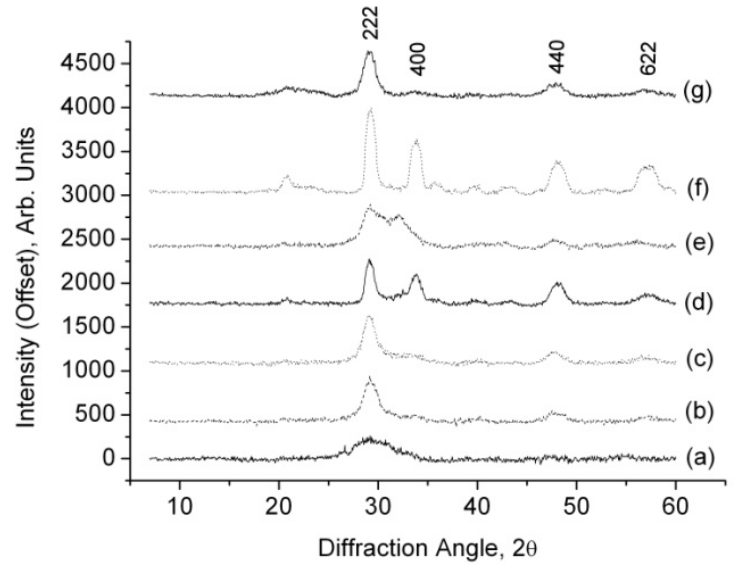

Fig. 2. XRD patterns at an incidence angle of $2^{\circ}$ for the $\mathrm{Y}_{2} \mathrm{O}_{3}$ thin films deposited using different parameters.

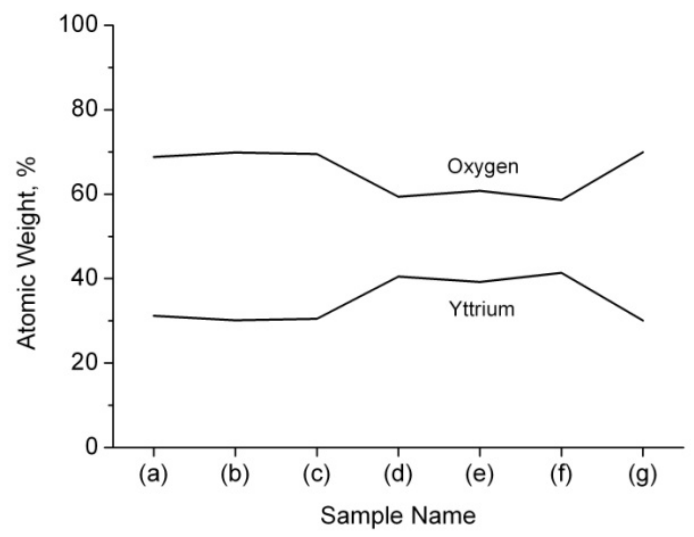

Fig. 3. Thin film composition for each sample measured using EDX. 

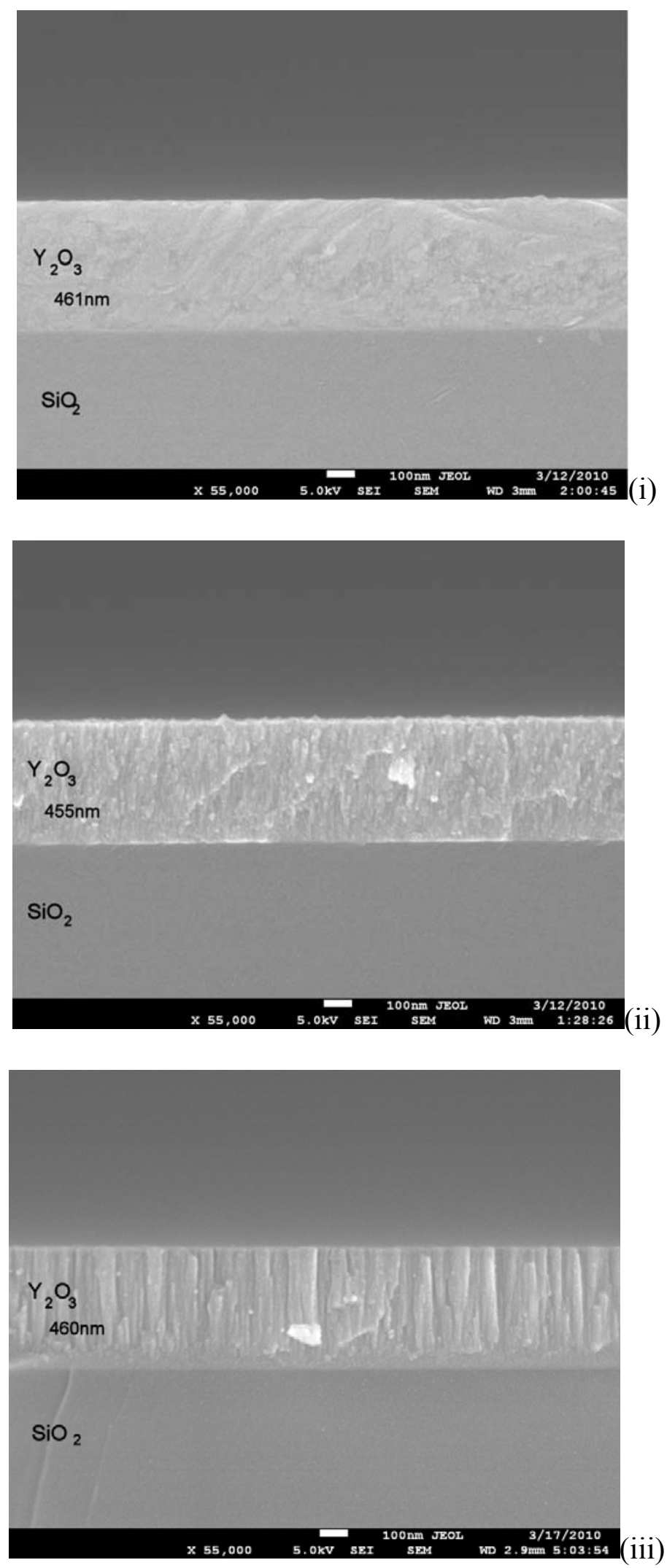

Fig. 4. SEM photographs of $\mathrm{Y}_{2} \mathrm{O}_{3}$ thin film layers (i) sample (b), (ii) sample (e) and (iii) sample (f). 